\title{
Reconstrucción dentoalveolar con implantes dentales mediante la técnica de levantamiento de seno maxilar
}

\author{
Dentoalveolar reconstruction with dental implants using the technique of maxillary sinus lifting \\ Luis Granados-Páucar ${ }^{1 a}$, María Cristina Ikeda-Artacho ${ }^{1 b c}$, Luis Sueldo-Gálvez ${ }^{1 b}$.
}

\section{RESUMEN}

La colocación de implantes en el sector posterosuperior con frecuencia debe enfrentar casos de atrofia de reborde maxilar y neumatización del seno maxilar, para los cuales una de las técnicas hoy en día empleada es la elevación del piso del seno maxilar mediante el aumento vertical del piso antral. Se reporta el caso de un paciente femenino de 60 años con exodoncias previas, diagnóstico de edentulismo parcial bimaxilar y neumatización del seno maxilar derecho. Se realizó como tratamiento el levantamiento del seno maxilar y la colocación de implantes a nivel postero superior izquierdo empleando la técnica mencionada. Se describe y documenta la premedicación quirúrgica, el momento operatorio, postoperatorio y el seguimiento exitoso del caso luego de seis meses del tratamiento.

PALABRAS CLAVE: Seno maxilar, cirugía, implante dental, reconstrucción dentoalveolar, levantamiento de seno maxilar, aumento de piso de seno maxilar.

\section{SUMMARY}

The placement of implants in the posterior superior areaof the mouth often has to face cases of maxillary ridge atrophy and pneumatization of the maxillary sinus, for which one of the techniques actually used is the elevation of the maxillary sinus floor by vertical increase of the antral floor. It is reported the case of a female patient of 60 years old with previous extractions, bimaxillarypartial edentulism diagnosis and pneumatization of the right maxillary sinus. Treatment was performed at the left posterior superior area for sinus lift and implant placement using the above technique. It is described and documented surgical premedication, surgical

\footnotetext{
${ }^{1}$ Facultad de Estomatología, Universidad Peruana Cayetano Heredia. Lima, Perú

a Docente.

Especialidades en Cirugía Bucal y Periodoncia.

c Doctorado en Estomatología.

c Especialidad en Periodoncia.
} 
approach, postoperative conditions and the successful follow up of the case after six months of the theraphy.

KEY WORDS: Maxillary sinus, surgery, dental implant, dentoalveolar reconstruction, sinus lift, sinus floor augmentation.

\section{INTRODUCCIÓN}

La instalación de implantes dentales en el sector postero superior de la cavidad bucal con frecuencia se ve complicada por la atrofia del reborde maxilar '10

Las técnicas hoy en día empleadas en la colocación de implantes sobre rebordes atróficos del sector posterosuperior consideran, desde el uso de implantes de menor longitud y de mayor diámetro, hasta tratamientos quirúrgicos como el levantamiento del seno maxilar o, más precisamente, la elevación del piso del seno maxilar mediante el aumento vertical del piso antral (1-3).

La técnica de levantamiento del seno maxilar fue inicialmente descrita en 1977 por Tatum, a partir de un acceso crestal mediante osteotomía (4). Es un método indirecto de abordaje del seno - conocido también como técnica a seno cerrado -que fue perfeccionado por R Summers, quien propuso el uso de osteóstomos de punta roma (5). Por otro lado, en 1980 P Boyne y $\mathrm{R}$ James emplearon el abordaje por la pared lateral del seno (antrostomía) (6). Desde entonces, se han presentado numerosas revisiones de ambas técnicas quirúrgicas comparando su eficacia y evidenciándose éxito clínico para ambos procedimientos (7-9).

Una revisión de la literatura muestra también el uso de diferentes tipos de biomateriales para el relleno del seno maxilar (10-16).

La elevación del piso del seno maxilar permite crear suficiente cantidad y calidad de hueso, de modo tal que, al aumentar el volumen de altura de la cresta ósea alveolar por su base, permita la estabilidad primaria requerida para la colocación de implantes (17).

La antrostomía tiene dos variantes: (a) La técnica en dos pasos, en la que se colocan los implantes después de 3 a 8 meses de realizada la cirugía de levantamiento de seno maxilar (6) y, (b) la técnica de un solo paso, en la cual se colocan los implantes en el mismo acto quirúrgico (18). La decisión depende del espesor del hueso inferior al piso del seno maxilar, según la recomendación presentada por Hunt y Jovanovic (Tabla 1)(19).

Estos estudios señalan que la osteotomía se recomienda cuando existe más de $6 \mathrm{~mm}$.de hueso residual y se requiera de hasta 3-4 mm.de incremento. En los casos de reabsorción más avanzada donde se requiera formar mayor espesor óseo, se prefiere la antrostomía de uno o dos pasos, de acuerdo a lo arriba descrito.

El levantamiento del seno maxilar mediante abordaje lateral es la técnica que permite lograr los aumentos más importantes en altura ósea. Esta técnica consiste en llevar parte de la cortical vestibular del seno maxilar hacia la zona oclusal del mismo, con el propósito de convertirla en el nuevo piso del seno maxilar y obtener mayor tamaño vertical de reborde maxilar. Ello contribuye a asegurar la estabilidad primaria del implante, con la posibilidad de que sea de mayor longitud (21-23).

Tabla 1. Clasificación del espesor del hueso inferior al piso del seno maxilar, según Jovanovic y Hunt.

\begin{tabular}{ll}
\hline Espesor del piso del seno maxilar & Colocación de los implantes \\
\hline Clase D $(0-3 \mathrm{~mm})$. & Diferida \\
Clase C $(4-6 \mathrm{~mm})$. & Diferida o Simultánea \\
Clase B $(7-9 \mathrm{~mm})$. & Simultánea \\
Clase A $(>10 \mathrm{~mm})$. & Simultánea \\
\hline
\end{tabular}




\section{REPORTE DE CASO}

Paciente de 60 años de edad, sexo femenino, en buen estado de salud general. Refiere haberse realizado con anterioridad las exodoncias de las piezas ausentes en ambos maxilares, sin complicaciones.

Al examen clínico intrabucal se verifica ausencia de piezas dentarias 1.6, 2.4-2.7, 3.6, 3.7, 4.4, 4.6 y 4.7.

Los exámenes auxiliares de laboratorio indicados fueron hemograma completo, perfil de coagulación, perfil lipídico, dosaje de glucosa, pruebas de VIH y VDRL; sus resultados fueron no contribuyentes.

El estudio imaginológico incluyó seriada radiográfica, tomografía (Figuras 1, 2, y 3) y fotografías clínicas. En la figura 1 la tomografía helicoidal muestra los cortes a nivel de los implantes a colocar. La figura 3 muestra una imagen radiopaca a nivel del seno maxilar izquierdo, que motivó una interconsulta con la especialidad de otorrinolaringología a fin de descartar sinusitis. La radiografía panorámica de la fig-
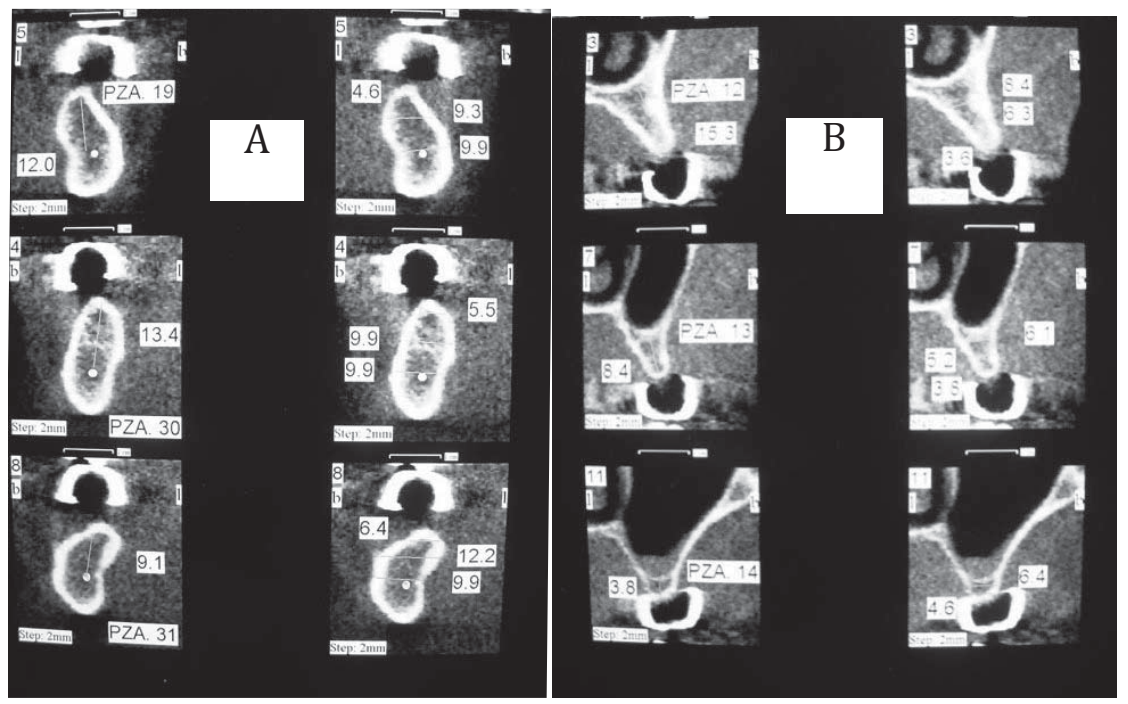

Figura 1. Tomografía helicoidal con cortes a nivel de los implantes a colocar.

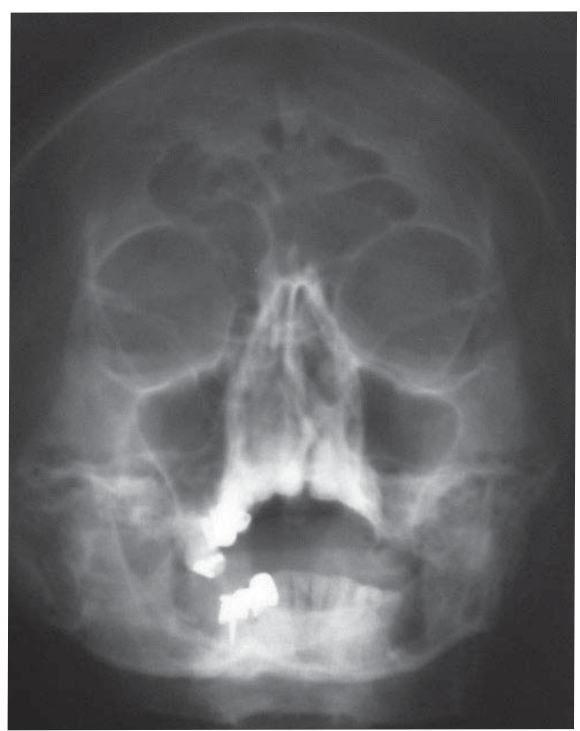

Figura 2. Imagen radiopaca a nivel del seno maxilar izquierdo, que motivó una interconsulta con la especialidad de otorrinolaringología.

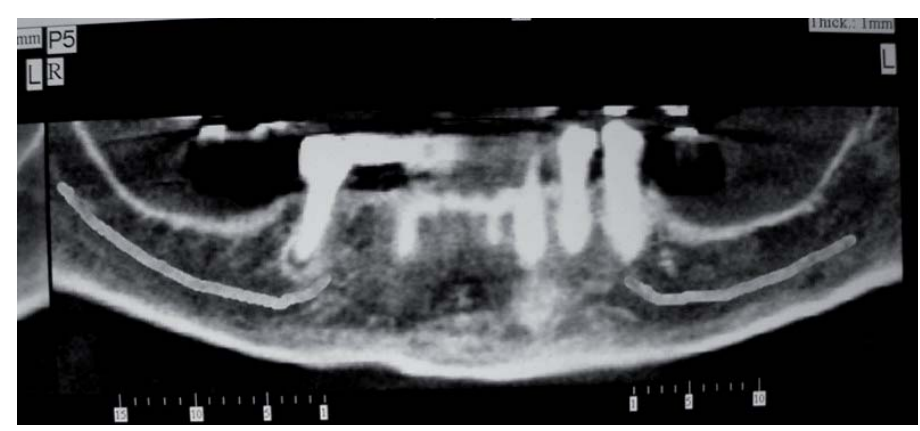

Figura 3. Corte panorámico mostrando posición del nervio dentario inferior en ambos lados. 
ura 1d indica la posición del nervio dentario inferior de ambos lados, contribuyendo a garantizar una instalación de implantes más segura.

El diagnóstico del caso consideró edentulismo parcial bimaxilar y neumatización de seno maxilar derecho, por lo cual se estableció como plan de tratamiento el levantamiento del seno maxilar, la colocación de implantes a nivel posterosuperior izquierdo y la colocación de implantes bilaterales en maxilar inferior.

En la fase preoperatoria se prescribió Dexametasona 4 mg.y Keterolaco 60 mg., vía intramuscular. A nivel local se realizó un enjuagatorio con gluconato de clorhexidinaal $0,12 \%$ como protocolo previo al abordaje quirúrgico.

El acto operatorio se llevó a cabo bajo anestesia

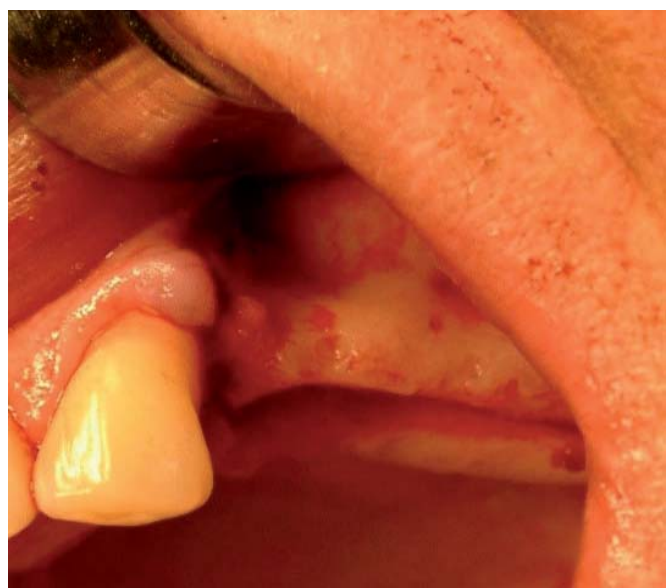

Figura 4. Levantamiento de colgajo a nivel de piezas posteriores y de seno maxilar.

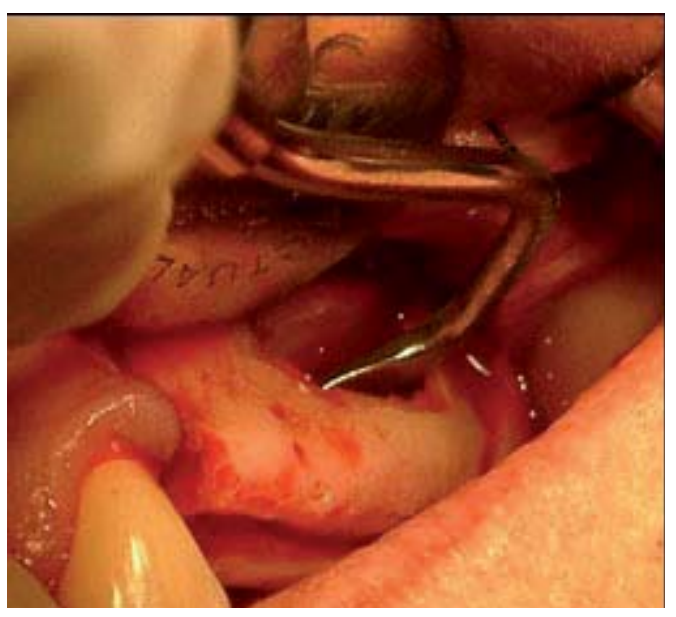

Figura 6. Pared vestibular del seno maxilar convertida en nuevo piso del seno; decolado de la membrana schneideriana. local infiltrativa a nivel de las piezas posteriores del cuadrante superior derecho. Se empleó una incisión paracrestal del reborde alveolar a nivel de piezas 2.42.7.Las figuras 4, 5 y 6 muestran el levantamiento del seno maxilar izquierdo. La figura 5 muestra la preparación de una ventana a nivel vestibular del seno maxilar izquierdo, la cual se convierte en nuevo piso del seno en la figura 6, junto al decolado de la membrana schneideriana.

La secuencia de colocación de implantes a nivel de piezas 2.4, 2.5 y 2.6 se muestra en las figuras 4, 5, 6, 7 y 8. La figura 7 corresponde a la preparación de sitios receptores de implantes, considerando la búsqueda de paralelismo entre estos. En la figura 8 se aprecia la instalación inicial de forma parcial de los implantes, enterrando solo la porción incluida en hueso. A continuación, en la figura 9 el seno maxilar

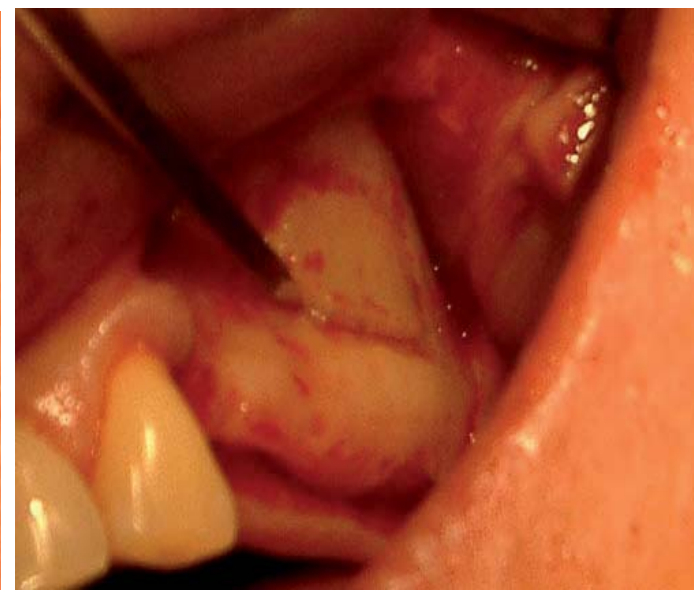

Figura 5. Ventana a nivel de pared de seno maxilar.

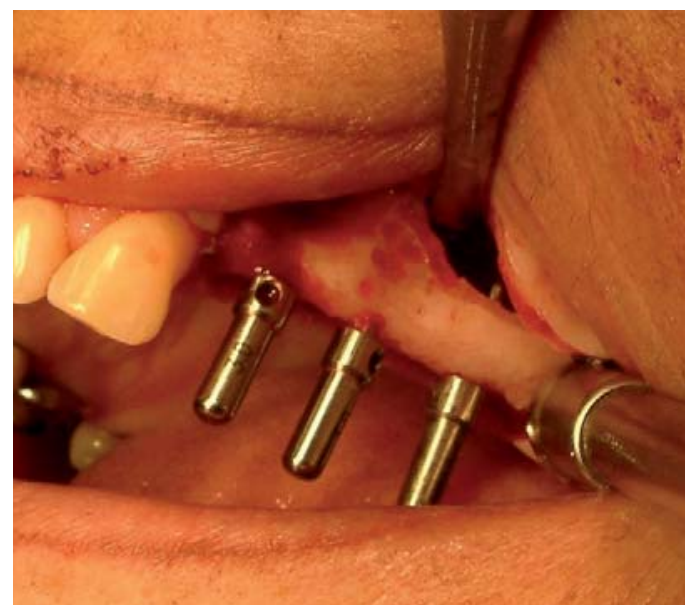

Figura 7. Preparación de sitios receptores de implantes; búsqueda de paralelismo entre los implantes. 


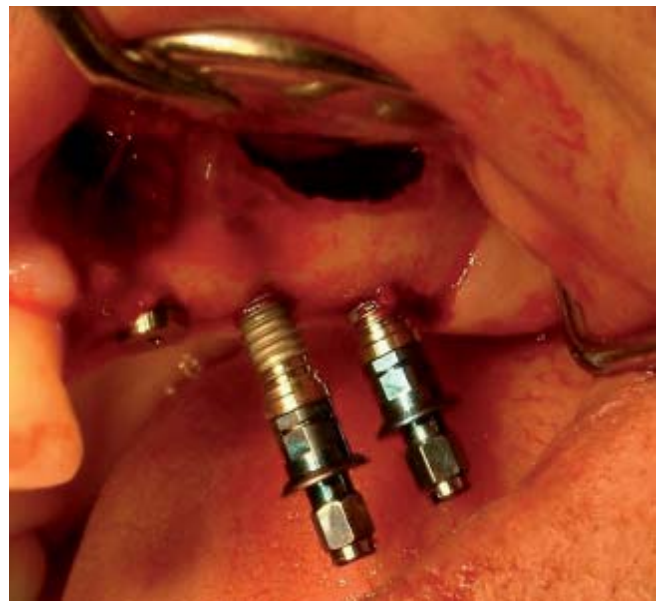

Figura 8. Instalación parcial de los implantes.Se entierra solo la porción incluida en hueso.

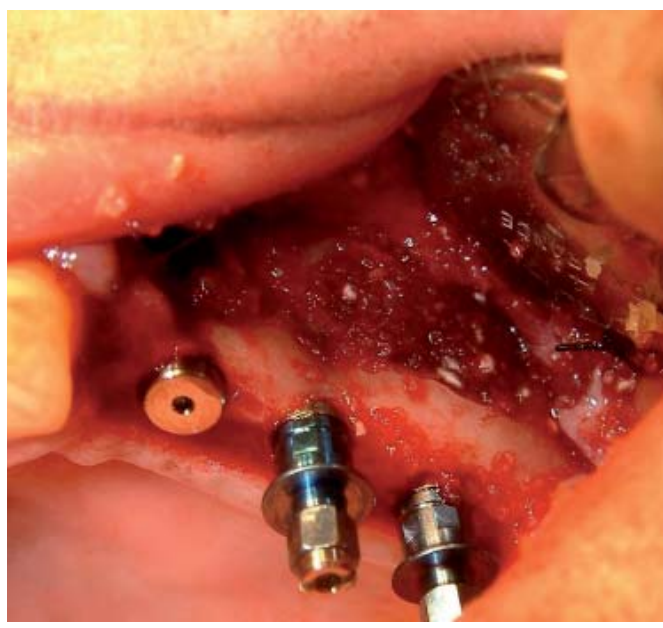

Figura 10. Instalación completa de los implantes y aumento de relleno para cubrir el defecto del seno maxilar.

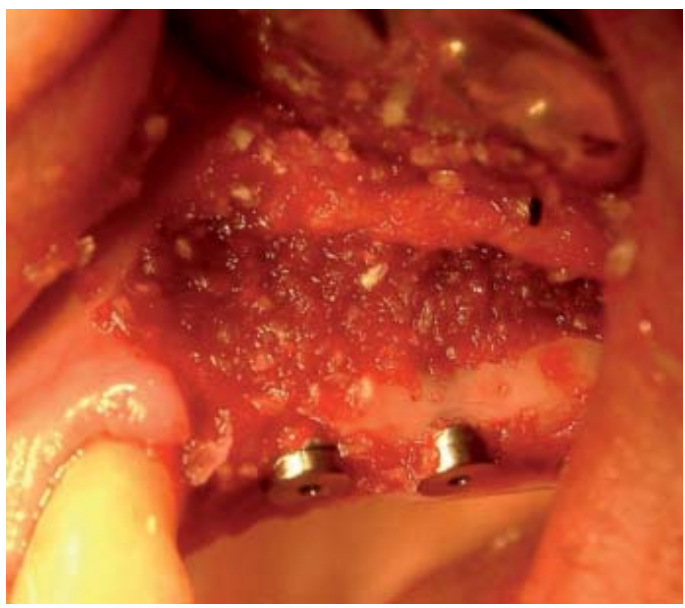

Figura 9. Después de rellenar el seno maxilar, se termina de enterrar los implantes.

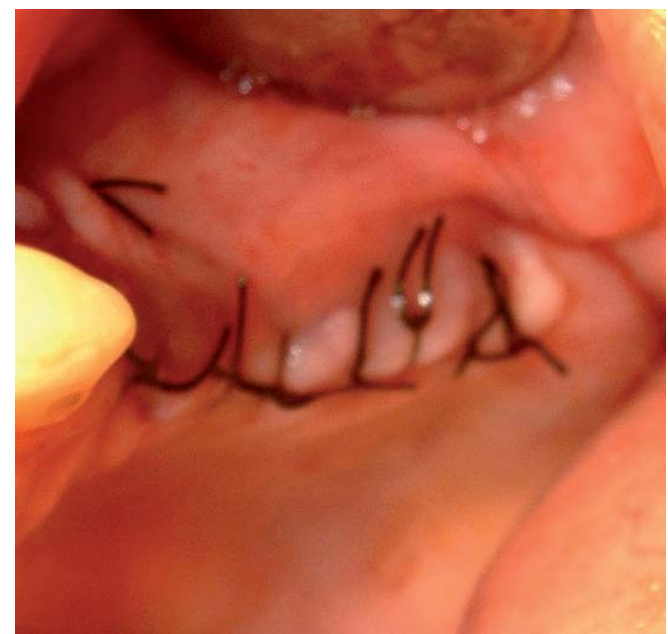

Figura 11. Sutura de los colgajos vestibular a palatino.

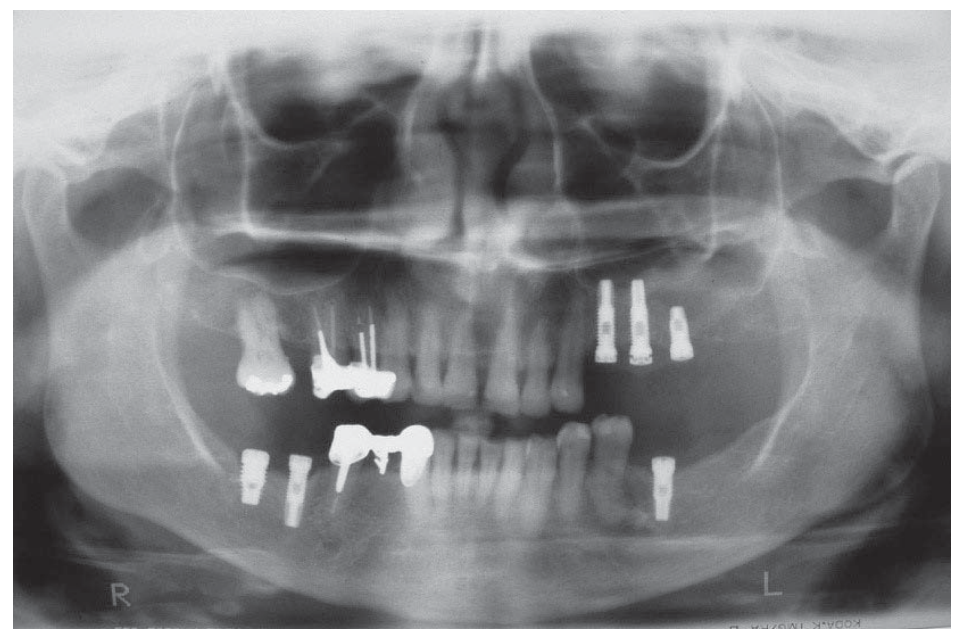

Figura 12. Control radiográfico a los seis meses. 
es rellenado con hueso artificial para, finalmente, terminar de enterrar los implantes. La figura 10 muestra la instalación completa de los implantes superiores y el aumento de relleno óseo para cubrir el defecto del seno maxilar. La cirugía superior concluye con la sutura del colgajo, de vestibular a palatino (Figura 11).

Un decolaje inferior permitió también la colocación de los implantes a nivel de las piezas 3.6, 4.6 y 4.7 .

La figura 12 corresponde a un control radiográfico realizado a los 6 meses de colocados los implantes.

La medicación postoperatoria consistió en 500 mg. de amoxicilina vía oral cada8 horas por 10 días, $4 \mathrm{mg}$. de dexametasona y $60 \mathrm{mg}$. de ketorolacovía intramuscular cada 12 horas por 2 días y, a partir del tercer día, $20 \mathrm{mg}$. de ketorolaco tabletas vía oral condicionado al dolor.Asimismo, se acompañó un control químico de placa con la prescripción de enjuagatorios de gluconato de clorhexidinaal 0,12\% a partir del tercer día postoperatorio durante 7 días. Las indicaciones postoperatorias incluyeron dieta blanda libre de cítricos, ají y alimentos condimentados, así como reposo físico por 48 horas.

Seis meses después del tratamiento quirúrgico, la evaluación clínica y la evaluación radiográfica de la figura 12 evidencian formación ósea a nivel del seno maxilar y osteointegración de los implantes. Se verificó ausencia de movilidad patológica, dolor, pérdida periimplantaria menor a $1,5 \mathrm{~mm}$., así como ausencia de sangrado y supuración de los tejidos blandos periimplantarios.

\section{DISCUSION}

La pérdida de altura vertical del hueso y la neumatización del seno maxilar muchas veces limita la colocación de implantes dentales en la zona posterosuperior de la boca.La literatura ofrece varias técnicas quirúrgicas empleadas con la finalidad de abordar esta zona anatómica y aumentar la altura de hueso disponible para la colocación de los implantes. El reporte de caso corresponde al uso de la técnica de aumento vertical del piso antral a partir del abordaje por la pared lateral del seno maxilar (antrostomía).
De acuerdo al 6th.EuropeanWorkshoponPeriodontology (2008), las complicaciones más frecuentes del aumento de piso antral son perforación de la membrana sinusal, infección del injerto y pérdida ósea capaz de inhabilitar la colocación de implantes (24); ninguna de estas se reporta para este caso clínico.

Este consenso señala también que cuando la altura del hueso residual es mayor a $6 \mathrm{~mm}$, un $17 \%$ de los sujetos sometidos a aumento de hueso mediante el abordaje lateral experimentan pérdida del implante en los 3 primeros años (24); este caso presenta un espesor de hueso del piso del seno maxilar de hasta $6 \mathrm{~mm}$.

La más reciente evaluación del caso corresponde a los seis meses (Figura 12), verificándose que cumple con los criterios de éxito más frecuentemente utilizados para evaluar la fase quirúrgica de colocación de implantes según lo descrito en una revisión sistemática realizada el 2012 por Papaspyridakos y col., que incluye: en el nivel implantario, ausencia de movilidad, dolor, radiolucidez y pérdida ósea periimplantariade hasta $1,5 \mathrm{~mm}$.; y, en el nivel de los tejidos blandos periimplantarios, ausencia de sangrado y supuración (25). Sin embargo, queda por reportar la evaluación a nivel técnico de la rehabilitación, así como la función y estética lograda luego de la misma; incluso actualmente la satisfacción y confort del paciente vienen siendo considerados como criterios de éxito del tratamiento utilizando el complejo implantario-rehabilitador. Se considera también las conclusiones del Proceedings of the 3rd. European Workshop on Periodontology (26), en donde se especifica que el implante no debe provocar reacción alérgica, tóxica o infecciosa, ofrezca soporte para una rehabilitación funcional, no muestre signos de fractura o incurvación, ni movilidad a la exploración manual, ni radiolucidez a la evaluación radiográfica; así como los criterios expuestos por Sáenz el 2013 (27).

\section{CONCLUSIONES}

La formación ósea, oseintegración y condiciones de tejidos blandos alcanzadas a los seis meses de colocación de los implantes cumplen con los criterios de éxito utilizados en la actualidad para la evaluación de los implantes en su fase quirúrgica (24-28). Este reporte de caso corresponde a un caso exitoso de uso de la técnica de levantamiento de seno maxilar y lare- 
construcción alveolar con implantes en el maxilar superior mediante el abordaje lateral. Se requiere mayor tiempo de seguimiento del caso para la evaluación del complejo implanto-rehabilitador.

\section{Correspondencia:}

\author{
María Cristina Ikeda Artacho \\ Correo electrónico: maria.ikeda@upch.pe
}

\section{REFERENCIAS BIBLIOGRÁFICAS}

1. Pagliai A. Elevación del piso del seno maxilar. Paris, Diplome D'Université D'Implantologie Orale et Maxillo-Faciale. 20002001. p. 32-43.

2. Tiwana PS, Kushner GM, Haug RH.. Maxillary sinus augmentation. Dent Clin North Am. 2006; 50(3): 409-24.

3. Palma VC, Magro-Filho O, de Oliveria JA, Lundgren S, Salata LA, Sennerby L. Bone reformation and implant integration following maxillary sinus membrane elevation: an experimental study in primates. Clin Implant Dent Relat Res. 2006; 8(1):11-24.

4. Tatum H Jr. Maxillary and sinus implant reconstructions. Dental Clinics of North America. 1986; 30(2): 207-229.

5. Summers R. A new concept in maxillary implant surgery: The osteotome technique. Compend Contin Educ Dent. 1994;15:152-60.

6. Boyne PJ, James RA. Grafting of the maxillary sinus floor with autogenous marrow and bone. Journal of Oral Surgery. 1980; 38(8):613-616.

7. Tetsch P, Lysek DA. Long-term results after lateral and osteotome technique sinus floor elevation: a retrospective analysis of 2190 implants over a time period of 15 years. Clinical Oral Implants Research. 2010; 21(5):497-503.

8. Esposito M, Grusovin MG, Rees J, et al. Interventions for replacing missing teeth: augmentation procedures of the maxillary sinus. Cochrane Database Syst Rev. 2010;(3):CD008397.

9. Pal US, Sharma NK, Singh RK, et al. Direct vs. indirect sinus lift procedure: A comparison. Natl J Maxillofac Surg. 2012;3(1):31-7.

10. Triplett RG, Nevins M, Marx RE, et al. Pivotal, randomized, parallel evaluation of recombinant human bone morphogenetic protein-2/absorbable collagen sponge and autogenous bone graft for maxillary sinus floor augmentation. Journal of Oral and Maxillofacial Surgery. 2009; 67(9):1947-1960.

11. Voss P, Sauerbier S, Wiedmann-Al-Ahmad M, et al. Bone regeneration in sinus lifts: comparing tissue-engineered bone and iliac bone. British Journal of Oral and Maxillofacial Surgery. 2010; 48(2):121-126.

12. Marx RE, Carlson ER, Eichstaedt RM, Schimmele SR, Strauss JE, Georgeff KR. Platelet-rich plasma: growth factor enhancement for bone grafts. Oral Surgery, Oral Medicine, Oral Pathology, Oral Radiology, and Endodontics. 1998; 85(6):638-646

13. Cordioli G, Mazzocco C, Schepers E, Brugnolo E, Majzoub Z. Maxillary sinus floor augmentation using bioactive glass granules and autogenous bone bone with simultaneous implant placement. Clin Oral Implants Res. 2001; 12(3):270-8.

14. Si MS, Zhuang LF, Gu YX, Mo JJ, Qiao SC, Lai HC. Osteotome sinus floor elevation with or without grafting: a 3-year randomized controlled clinical trial. J Clin Periodontol. 2013; 40(4):396-403.

15. Mazor Z, Horowitz RA, Del Corso M, Prasad HS, Rohrer MD, Dohan Ehrenfest DM. Sinus floor augmentation with simultaneous im- plant placement using Choukroun's platelet-rich fibrin as the sole grafting material: a radiologic and histologic study at 6 months. J Periodontol. 2009; 80(12):2056-64.

16. Riben $\mathrm{Ch}$, Thor A. The maxillary sinus membrane elevation procedure: Augmentation of bone around dental implants without grafts, a review of surgical technique. Int J Dent. 2012; 2012:105483.

17. Dedigi M, Daprile G, Piattelli A. Primary stability determination of implants inserted in sinus augmented sites: 1 -step versus 2 -step procedure. Implant Dent. 2013; 22(5):530-3.

18. Hirsch JM, Ericsson I. Maxillary sinus augmentation using mandibular bone grafts and simultaneous installation of implants. A surgical technique. Clin Oral Implants Res. 1991; 2(2):91-6.

19. Hunt DR, Jovanovic SA. Autogenous bone harvesting: A chin graft o technique for particulate and monocortical bone blocks. Int J Periodontics Restaurative Dent 1999;19:165-73.

20. Pjetursson BE, Tan WC, Zwahlen M, Lang NP. A systematic review of the success ofsinus floor elevation and survival of implants inserted in combination with sinus floor elevation. Part I: Lateral approach. J ClinPeriodontol. 2008; 35 (S8):216-240.

21. WahChing T, Niklaus L, Zwahlen M, Bjarni P. A systematic review of the success of sinus floor elevation and survival of implants inserted in combination with sinus floor elevation Part II: Transalveolar technique. J Clin Periodontol. 2008; 35 (S8):241-254.

22. Kahnberg KE, Ekestubbe A, Gröndahl K, Nilsson P, Hirsch JM. Sinus lifting procedure. I. One-stage surgery with bone transplant and implants. Clin Oral Implants Res. 2001; 12(5):479-87.

23. Antonaya-Mira R, Barona-Dorado C, Martínez-Rodríguez N, Cáceres-Madroño E, Martínez-González JM. Meta-analysis of the increase in height in maxillary sinus elevations with osteotome. Med Oral Patol Oral Cir Bucal. 2012; 17(1): e146-e152.

24. Tonetti MS, Hammerle CHF. Advances in bone augmentation to enable dental implant placement: Consensus Report of the Sixth European Workshop on Periodontology. J Clin Periodontol. 2008; 35(S8): 168-72.

25. Papaspyridakos P, Chen C-J, Singh M, Weber H-P, Gallucci GO. Success criteria in implant dentistry: A systematic review. J Dent Res. 2012; 91(3):242-8.

26. Ismail J. Proceedings of the Third European Workshop on Periodontology: Implant Dentistry. Journal of Prosthodontics. 2001; 10(4):275-276.

27. Saenz M. Criterios de éxito y fracaso en implantes dentales oseointegrados. Acta Odontológica Venezolana. 2013; 51(2):18.

28. Papaspyridakos P, Chen C-J, Chuang S-K, Weber H-P, Gallucci GO. Systematic review of biologic and technical complications with fixed implant rehabilitations for edentulous patients. Int J Oral Maxillofac Implants. 2012;27:102-110.

Recibido : 03/05/2014

Aceptado: 10/08/2014 